ARTIGO ORIGINAL ORIGINAL ARTICLE

\title{
Vinculação aos planos de saúde de menor abrangência e seu impacto na utilização da rede de serviços do SUS: uma análise a partir da Pesquisa Nacional de Saúde de 2013
}

\author{
Linkage to health plans of lesser extent and their \\ impact on the use of the SUS service network: an \\ analysis from the National Health Survey of 2013 \\ Carlos Gomide da Silva', Ronir Raggio Luiz², Ligia Bahia³ \\ DOI: 10.21115/JBES.v11.n2.p135-41
}

\section{Palavras-chave:}

sistemas de saúde privados, Sistema Único de Saúde, Pesquisa Nacional de Saúde

\section{Keywords:}

private health systems, Unified Health System, National Health Survey

\section{RESUMO}

Objetivo: Identificar se a vinculação aos referidos planos pode ser considerada como uma causa ou um fator incrementador da utilização do SUS por esses clientes. Métodos: Trabalhou-se com os dados fornecidos pela Pesquisa Nacional de Saúde realizada em 2013. A técnica utilizada foi a do escore de propensão. Resultados: Houve significância estatística quando se avaliou a utilização do Sistema Único de Saúde (SUS) pelos clientes dos planos de menor abrangência tanto em consultas e outros procedimentos quanto para internações, sendo essas utilizações, respectivamente, de 37,9\% e 46,4\%. Conclusão: Pode-se observar que a vinculação aos planos de menor abrangência se constitui em um fator incrementador à utilização da rede de serviços do SUS, reforçando a argumentação de que a oferta desses sistemas protetivos no mercado não contribui para desafogar a rede pública de saúde.

\section{ABSTRACT}

Objective: To identify whether the link to these plans can be considered as a cause or an incremental factor of the use of SUS by these clients. Methods: Data from the National Health Survey conducted in 2013 were used. The technique used was the propensity score. Results: Statistical significance was found when the use of the Unified Health System (SUS) was evaluated by the clients of the plans of lesser extent in consultations and other procedures, as well as hospitalizations, $37.9 \%$ and $46.4 \%$, respectively. Conclusion: It can be observed that the linkage to the less comprehensive plans is an incremental factor for the use of the SUS service network, reinforcing the argument that the supply of these protective systems in the market does not contribute to the public health network.
Recebido em: 29/05/2019. Aprovado para publicação em: 24/08/2019

1. Professor do Departamento de Economia, Universidade Federal de Viçosa, Viçosa, MG, Brasil.

2. Professor do Instituto de Estudos em Saúde Coletiva, Universidade Federal do Rio de Janeiro, Rio de Janeiro, RJ, Brasil.

3. Professora do Instituto de Estudos em Saúde Coletiva, Universidade Federal do Rio de Janeiro, Rio de Janeiro, RJ, Brasil.

Nome da instituição onde o trabalho foi executado: Programa de Pós-Graduação do Instituto de Estudos em Saúde Coletiva (IESC) da Universidade Federal do Rio de Janeiro (UFRJ).

Informações sobre auxílios recebidos sob a forma de financiamento, equipamentos ou medicamentos - congressos onde o estudo foi apresentado: Não houve auxílio financeiro. Este trabalho é inédito resultante da tese de doutorado em Saúde Coletiva de Carlos Gomide da Silva.

Autor correspondente: Carlos Gomide da Silva. Rua Padre João Silvestre, 455, Centro, Teixeiras, MG, Brasil. CEP: $36580-000$. Telefone: (31) 99761-2806. E-mail: cgsilva@ufv.br 


\section{Introdução}

A Constituição da República de 1988 estabeleceu como direito de cidadania o acesso e a utilização dos serviços em saúde a toda a população, que passa, a partir de então, a ter direito de utilizar o novo sistema público de proteção à saúde denominado Sistema Único de Saúde (SUS). A Carta Constitucional também ratificou a permanência dos sistemas privados de proteção à saúde que já existiam anteriormente, bem como possibilitou a entrada de novas empresas atuando nesse mercado (Brasil, 1988).

Apesar dos avanços, o acesso e a utilização dos serviços em saúde ainda não se tornaram equitativos no Brasil. Há diferenças marcantes segundo a classe socioeconômica, e os extratos da população com menor renda ainda apresentam baixas taxas de utilização de serviços especializados, evidenciando as desigualdades entre as classes. Essas desigualdades no acesso e na utilização, além de ocorrerem entre as pessoas vinculadas e não vinculadas aos planos de saúde, também são perceptíveis entre os clientes dos planos, de tal modo que essa clientela, em um contexto de maior renda, se beneficia de melhor acesso aos cuidados em saúde (Viana et al., 2015).

Existem mais de mil operadoras de planos e seguros de saúde, entretanto a maior parte da clientela está concentrada em poucas delas. São disponibilizados diferentes tipos de planos, que abarcam mais de 47 milhões de clientes no país (Brasil-ANS, 2018). Ocorre variação também na distribuição dessa cobertura entre os estados e municípios, concentrando-se em menos de $30 \%$ deles, contribuindo para a dificuldade de acesso e de utilização dos serviços em saúde pelas pessoas residentes nos locais onde a oferta é mais escassa ou inexistente (Santos et al., 2008; Bahia et al., 2014).

A vinculação dos clientes aos planos é distribuída em dois grandes grupos de esquemas assistenciais: sistema de proteção à saúde dos servidores municipais, estaduais e militares e o grupo formado pelos sistemas de proteção à saúde ofertados pelas empresas privadas a empresas empregadoras e às famílias. Este último ainda pode ser segmentado em diferentes modalidades de sistemas protetivos, tipificados em: seguradoras especializadas em saúde, cooperativas médicas, medicinas de grupo, sistemas de autogestão e redes de filantropia (Brasil-ANS, 2018).

O modo de adesão ao plano de saúde também varia. Quando o plano é vinculado ao emprego do titular, ele pode ser classificado como sendo um plano coletivo. Nessa modalidade, os valores de mensalidades podem ser divididos entre o titular e o empregador ou totalmente assumidas por esse último (plano coletivo empresarial). Quando o titular paga a mensalidade diretamente ao plano, esse plano é denominado individual ou familiar (Brasil, 2018). Ressalta-se que os planos também podem variar quanto à natureza em função do tipo de cobertura. Essa pode ser ambulatorial, visando ape- nas a consultas e exames, ou hospitalar, quando inclui a internação. Entretanto, o principal marcador de qualidade dos planos é o seu preço. Ainda que os planos comparados sejam considerados "tecnicamente equivalentes", haverá grande diferença no tratamento dispensado ao cliente em função do valor da mensalidade. Sendo assim, esse valor pago pode ser considerado uma proxy da abrangência do plano (Bahia et al., 2014).

O valor mensal desembolsado pode comprometer parte expressiva da renda das famílias, mas possibilita que as pessoas tenham o direito de receber atendimento na rede privada de saúde. No entanto, quando esses clientes utilizam o atendimento oferecido pelo SUS, isso evidencia, em qualquer nível de qualidade do plano contratado, a dificuldade de acesso e utilização desses sistemas. Essas dificuldades podem ocorrer por vários motivos, tais como: negações de cobertura por parte das operadoras (especialmente em procedimentos mais caros), fatores geográficos geralmente relacionados ao local de domicílio, além de outras barreiras. Ressalta-se que essa utilização da rede de serviços do SUS vem ocorrendo de modo crescente (Silva et al., 2011) e tem sido observada com maior frequência entre as pessoas que contrataram planos mais acessíveis, os quais são caracterizados principalmente por possuírem valores de mensalidades que são incompatíveis com o custo real dos procedimentos médicos, especialmente os de maior complexidade (Azevedo Junior et al., 2012).

Embora a Lei no 9.656/98, popularmente conhecida como "Lei dos Planos de Saúde", obrigue as operadoras a disponibilizarem cobertura total em saúde de acordo com as necessidades de sua clientela, isso constantemente não é observado na prática. A negação de cobertura por parte delas faz com que muitos clientes de planos de saúde sejam obrigados a recorrer à justiça com o intuito de fazer valer os seus direitos. Ressalta-se que um volume considerável desses processos está associado aos planos mais populares (Sestelo et al., 2014).

A pressão sobre os legisladores promovida pelas operadoras no intuito de obter a legalização de planos populares de cobertura limitada, consequentemente fora do alcance jurídico da Lei no 9.656/98, tem sido constante e intensificada nos períodos de maior recessão econômica. No entanto, os defensores da legalização dessa modalidade de plano argumentam que não há como obter mais recursos para a área da saúde dentro do governo. Além disso, estimativas do próprio governo federal apontam que $85 \%$ dos problemas de saúde da população poderiam ser resolvidos na atenção primária e, nesse caso, poderiam ser solucionados por planos que ofertassem basicamente os itens utilizados nesse tipo de atendimento. Sendo assim, esse tipo de plano desafogaria o SUS. Os procedimentos contemplados por esses planos seriam mais baratos e, consequentemente, permitiriam possuir valores de mensalidades mais compatíveis com o poder aquisitivo da maior parte da população, desonerando o SUS (Mendes, 2016). 
Em contrapartida, a legalização dessa modalidade de plano, que em verdade já vem sendo ofertada na prática, poderia resultar em piora da situação do SUS. Isso porque, ao se depararem com as limitações impostas pelo plano quando da necessidade de tratamentos de maior complexidade, o paciente seria redirecionado ao SUS, que o retornaria ao início do tratamento, provocando atrasos, já que não poderiam utilizar os encaminhamentos fornecidos pelo plano privado na rede pública. Assim sendo, tais planos não seriam capazes de aliviar a falta de recursos do SUS e nem agilizariam o atendimento para pacientes com doenças mais complexas. Ainda teriam efeito contrário, em virtude da desorganização que ocorreria no sistema de saúde (Scheffer \& Bahia, 2015).

Em se considerando o fato de que o vínculo a um plano mais barato, ou menos abrangente, está associado a uma maior utilização da rede de serviços do SUS por parte dos clientes desses planos (Scheffer, 2015), e que, possuindo essa associação um nexo causal, isso reforçaria a hipótese de que a legalização da oferta desses esquemas assistenciais se constituiria em um fator incrementador da utilização da rede de serviços do SUS por essa clientela, essa pesquisa teve por objetivo trazer mais elementos sobre esse cenário, identificando a natureza da associação entre o vínculo aos planos de saúde menos abrangentes e a maior utilização da rede de serviços do SUS por seus clientes.

\section{Métodos}

As informações utilizadas neste estudo foram obtidas no banco de dados da Pesquisa Nacional de Saúde (PNS) realizada em 2013. A PNS é um estudo seccional, de base domiciliar, de âmbito nacional, realizada por amostragem probabilística, que utiliza uma amostra selecionada em um plano amostral complexo. A PNS também considera conglomeração das unidades em um, dois ou três estágios de seleção, além da estratificação das unidades definidas separadamente por unidade da federação (Souza-Júnior et al., 2015).

O total de vínculos aos planos de saúde individuais foi dividido em dois grupos. Primeiro, os que buscaram atendimento em saúde nas últimas duas semanas em consultas e outros procedimentos. O segundo grupo foi constituído pelos clientes desses planos que estiveram internados por 24 horas ou mais nos últimos 12 meses. Em cada grupo, foram discriminados os clientes vinculados aos planos de menor abrangência (planos individuais com mensalidades iguais ou inferiores a R\$300,00, doravante denominados por PI-B) daqueles vinculados aos planos mais abrangentes (planos com mensalidades superiores a $\mathrm{R} \$ 300,00$, doravante denominados por PI-A).

O valor de R\$300,00 (trezentos reais) foi estabelecido considerando-se os custos apresentados na Tabela Única Nacional de Equivalência de Procedimentos (TUNEP) obtidos no site da Agência Nacional de Saúde - ANS (Brasil-ANS, 2018), que apresenta os valores médios dos procedimentos médicos reembolsados pelo SUS. Também foram considerados para essa estimativa os valores de mensalidades existentes como opções de resposta no questionário da PNS para os planos individuais no ano de 2013 (IBGE, 2014).

Para controlar o viés pelo confundimento, recorrente em estudos seccionais, utilizou-se a técnica do escore de propensão com estratificação pelos quintis. O modelo utilizado neste trabalho para avaliar a existência de uma relação de causa e efeito, baseou-se na lógica do contrafato (Luiz \& Struchiner, 2002). A análise contrafactual foi adotada para a exposição, caracterizada pelo vínculo dos clientes a um dos dois tipos de planos de saúde individuais. O desfecho foi definido como sendo a variável binária "utilização (sim ou não) da rede de serviços do SUS".

Os escores foram determinados utilizando-se o modelo logístico binário, que, devido à configuração matemática adotada, permitiu que a exposição fosse apresentada em termos de probabilidades de vínculo aos dois tipos de planos individuais. Entre essas, optou-se por utilizar a probabilidade de vínculo ao plano individual com mensalidade igual ou inferior a $\mathrm{R} \$ 300,00$ (menor abrangência). A probabilidade de vínculo corresponde ao escore de propensão. A comparação do desfecho com a exposição foi efetuada em cada quintil. Posteriormente, esses resultados ajustados pelos quintis foram ponderados para determinar um percentual global de utilização do SUS, definindo um desfecho para consultas e outros procedimentos e outro para internações. As variáveis que definem os desfechos são encontradas no "Módulo J" do questionário da PNS 2013, que trata da utilização dos serviços de saúde.

O modelo matemático utilizado foi controlado pelas variáveis potencialmente confundidoras, por terem relação tanto com o desfecho quanto com a exposição (Hosmer \& Lemeshow, 2002). A escolha dessas variáveis levou em consideração características demográficas, socioeconômicas, geográficas, comportamentais e de saúde, que retratam características individuais e do ambiente em que os indivíduos se encontram inseridos.

Com o intuito de possibilitar a incorporação das informações do plano amostral complexo, além de viabilizar o emprego da técnica do escore de propensão, foi utilizado neste trabalho, em seu módulo complex sample, o software estatístico SPSS para Windows, versão 24.0 (SPSS Inc., Chicago, Estados Unidos).

\section{Resultados}

Resultados obtidos na PNS (PNS 2013) apontam que, dos mais de 40 milhões de clientes de planos de saúde maiores de 18 anos, cerca de 9 milhões buscaram atendimento em serviço de saúde nas duas últimas semanas. Entre esses, 15,0\% (IC de 95\%: $13,1 \%$ a 17,1\%) afirmaram que esse atendimento foi feito 
pelo SUS. No que tange às internações, cerca de 3,6 milhões de pessoas dessa população estiveram internadas por 24 horas ou mais nos últimos 12 meses. Desse montante, 17,0\% (IC de $95 \%:$ 13,8\% a 20,8\%) afirmaram que a internação foi feita pelo SUS.

Ao se considerar a população maior de 18 anos vinculada aos planos individuais, contabilizam-se mais de $10 \mathrm{mi}-$ Ihões de clientes. Isso representa 24,0\% (IC de 95\%: 22,6\% a 25,5\%) do total de vínculos aos planos de saúde privados e particulares. De acordo com os critérios estabelecidos nessa pesquisa, 52,9\% (IC de 95\%: 49,7\% a 56,2\%) desse total são vínculos aos planos considerados de menor abrangência (PI-B: mensalidades iguais ou inferiores a R\$ 300,00) e 47,1\% (IC de 95\%: 43,8\% a 50,3\%) o são aos planos considerados de maior abrangência (PI-A: mensalidades acima de R\$ 300,00).

Os resultados obtidos em análise bruta para a variável desfecho (utilização da rede de serviços do SUS) mostraram utilização significativamente maior dessa rede pelos clientes vinculados aos planos de menor abrangência.

Ao se proceder às análises utilizando a técnica do ajuste pelos quintis do escore de propensão, utilizaram-se as variáveis socioeconômicas, demográficas e de saúde apresentadas na Tabela 1, na qual são mostrados também os respectivos valores da estatística "F", antes e após o ajuste pelos quintis.

A viabilidade da utilização da técnica do escore de propensão pôde ser testada por meio da verificação da exis- tência da sobreposição (overlap) entre os indivíduos com probabilidades (ou escores iguais) de estarem vinculados a um tipo de plano, porém vinculados a planos distintos. Nesta pesquisa, o escore utilizado foi representado pela probabilidade de vínculo ao plano de menor abrangência (PI-B). A Figura 1, mostra a presença dessa faixa comum de clientes com a mesma probabilidade de vínculo ao plano PI-B, porém discriminando os que são vinculados, de fato, ao plano PI-B daqueles que o são aos planos de maior abrangência (PI-A) antes do ajuste pelos quintis. Ao se proceder às comparações utilizando-se a estratificação pelos quintis, o escore de propensão foi categorizado. Desse modo, no primeiro quintil, concentram-se os indivíduos com as mais baixas probabilidades de vínculo a um plano do tipo PI-B. No quinto quintil, em contrapartida, tem-se elevada concentração de indivíduos com grande probabilidade de vínculo a esse tipo de plano. Após o ajuste pelos quintis, pode-se observar, pela Figura 2, 0 efeito causado pelo balanceamento ocorrido dentro de cada subclasse da probabilidade estimada de vínculo ao plano de menor abrangência.

A comparação entre a variável desfecho - representada pela utilização da rede do SUS - e a exposição - representada pelo tipo de plano individual - em cada um dos quintis, para procedimentos e consultas nos últimos 15 dias e para as internações nos últimos 12 meses por 24 horas ou mais, estão representadas, respectivamente, nas Tabelas 2 e 3.

Tabela 1. Distribuição e comparação das variáveis socioeconômicas, geográficas, comportamentais e de saúde, dos clientes dos dois tipos de planos individuais e análise de variância (Estatística-F) antes e depois do controle pelos quintis dos escores de propensão

\begin{tabular}{|c|c|c|c|c|c|c|}
\hline \multirow[b]{3}{*}{ Covariáveis } & \multicolumn{4}{|c|}{ Plano de saúde individual } & \multirow{3}{*}{$\begin{array}{l}\text { Estatística-F } \\
\text { antes da } \\
\text { Estratificação }\end{array}$} & \multirow{3}{*}{$\begin{array}{l}\text { Estatística-F } \\
\text { depois da } \\
\text { Estratificação }\end{array}$} \\
\hline & \multicolumn{2}{|c|}{$\begin{array}{l}\text { PI-B: menor abran. } \\
\quad(n=5.629 .940)\end{array}$} & \multicolumn{2}{|c|}{$\begin{array}{l}\text { PI-A: maior abran. } \\
\qquad(n=5.002 .997)\end{array}$} & & \\
\hline & Média & Erro-padrão & Média & Erro-padrão & & \\
\hline Sexo $($ masculino $=1)$ & 0,39 & 0,019 & 0,47 & 0,019 & $8,54^{*}$ & 0,12 \\
\hline Idade (em anos) & 46,62 & 0,007 & 53,85 & 0,008 & $44,45^{*}$ & 0,41 \\
\hline Raça (branca = 1) & 0,62 & 0,018 & 0,78 & 0,016 & $41,81^{*}$ & 0,34 \\
\hline Condição de saúde (boa = 1) & 0,71 & 0,018 & 0,79 & 0,015 & $12,03^{*}$ & 0,03 \\
\hline Regiões (Sudeste = 1) & 0,56 & 0,017 & 0,68 & 0,020 & $5,57^{*}$ & 0,04 \\
\hline Doença crônica (sim = 1) & 0,29 & 0,018 & 0,31 & 0,018 & 1,07 & 0,04 \\
\hline Estado civil (casado = 1) & 0,49 & 0,019 & 0,62 & 0,018 & $23,49^{*}$ & 0,17 \\
\hline Escolarização (superior = 1) & 0,24 & 0,016 & 0,51 & 0,024 & $39,31^{*}$ & 0,04 \\
\hline Visita ag. endemias $(\operatorname{sim}=1)$ & 0,70 & 0,018 & 0,64 & 0,021 & 5,72 & 0,02 \\
\hline Dep. não morador $(\operatorname{sim}=1)$ & 0,12 & 0,012 & 0,09 & 0,012 & 3,99 & 0,17 \\
\hline Seg. saúde (serv. público = 1) & 0,10 & 0,011 & 0,06 & 0,009 & 6,53 & 0,13 \\
\hline Condição da moradia (boa = 1) & 0,77 & 0,016 & 0,88 & 0,013 & $29,73^{*}$ & 0,52 \\
\hline Renda média per capita (SM) & 1,12 & 0,001 & 1,37 & 0,001 & $10,18^{*}$ & 0,56 \\
\hline Total & 0,53 & 0,016 & 0,47 & 0,016 & - & - \\
\hline
\end{tabular}

Estimativas realizadas incorporando-se as características do plano amostral complexo da PNS-2013.

* Significância estatística para $p<0,05$.

Fonte: elaboração própria a partir dos microdados da PNS-2013. 
Tabela 2. Proporção de consultas e procedimentos médicos realizados na rede de serviços do SUS, nas últimas duas semanas, pelos clientes de planos de saúde de menor abrangência (PI-B) e de maior abrangência (PI-A), depois do ajuste pelo quintil (subclasses) do escore de propensão estimado, Pesquisa Nacional de Saúde (PNS-2013)

\begin{tabular}{|c|c|c|c|c|}
\hline Quintis & $\begin{array}{l}\text { Tipo de abrangência do } \\
\text { plano individual }\end{array}$ & Número de vínculos & $\begin{array}{l}\text { Utilização do SUS } \\
\text { em consultas e } \\
\text { procedimentos (\%) }\end{array}$ & Erro-padrão \\
\hline \multirow[t]{2}{*}{$1^{\circ}$} & $\mathrm{Pl}-\mathrm{B}$ & 31.895 & 24,0 & 0,185 \\
\hline & $\mathrm{Pl}-\mathrm{A}$ & 7.611 & 2,1 & 0,014 \\
\hline \multirow[t]{2}{*}{$2^{\circ}$} & PI-B & 59.607 & 27,1 & 0,083 \\
\hline & $\mathrm{Pl}-\mathrm{A}$ & 13.120 & 3,8 & 0,009 \\
\hline \multirow[t]{2}{*}{$3^{\circ}$} & PI-B & 113.170 & 32,6 & 0,077 \\
\hline & $\mathrm{Pl}-\mathrm{A}$ & 4.481 & 1,9 & 0,006 \\
\hline \multirow[t]{2}{*}{$4^{\circ}$} & $\mathrm{PI}-\mathrm{B}$ & 103.539 & 34,6 & 0,062 \\
\hline & $\mathrm{Pl}-\mathrm{A}$ & 6.494 & 4,6 & 0,022 \\
\hline \multirow[t]{2}{*}{$5^{\circ}$} & $\mathrm{PI}-\mathrm{B}$ & 184.464 & 48,9 & 0,051 \\
\hline & $\mathrm{Pl}-\mathrm{A}$ & 17.801 & 9,7 & 0,029 \\
\hline \multirow{2}{*}{$\begin{array}{l}\text { Total diretamente ajustado } \\
\text { entre os quintis }\end{array}$} & $\mathrm{PI}-\mathrm{B}$ & 492.675 & 37,9 & 0,053 \\
\hline & $\mathrm{Pl}-\mathrm{A}$ & 49.507 & 5,6 & 0,013 \\
\hline
\end{tabular}

Baseado no escore de propensão estimado.

Fonte: elaboração própria a partir dos microdados da PNS-2013.

Tabela 3. Proporção de internações realizadas na rede de serviços do SUS, nos últimos 12 meses por 24 horas ou mais, pelos clientes de planos de saúde de menor abrangência (PI-B) e de maior abrangência (PI-A), depois do ajuste pelo quintil (subclasses) do escore de propensão estimado, Pesquisa Nacional de Saúde (PNS-2013)

\begin{tabular}{|c|c|c|c|c|}
\hline Quintis & $\begin{array}{l}\text { Tipo de abrangência do } \\
\text { plano individual }\end{array}$ & Número de vínculos & $\begin{array}{l}\text { Utilização do SUS em } \\
\text { internações (\%) }\end{array}$ & Erro-padrão \\
\hline \multirow[t]{2}{*}{$1^{\circ}$} & $\mathrm{Pl}-\mathrm{B}$ & 2.018 & 11,4 & 0,112 \\
\hline & $\mathrm{PI}-\mathrm{A}$ & 2.453 & 2,7 & 0,027 \\
\hline \multirow[t]{2}{*}{$2^{\circ}$} & PI-B & 3.630 & 5,9 & 0,027 \\
\hline & $\mathrm{PI}-\mathrm{A}$ & 416 & 0,2 & 0,002 \\
\hline \multirow[t]{2}{*}{$3^{\circ}$} & $\mathrm{Pl}-\mathrm{B}$ & 59.989 & 50,2 & 0,054 \\
\hline & $\mathrm{PI}-\mathrm{A}$ & 8.116 & 9,2 & 0,055 \\
\hline \multirow[t]{2}{*}{$4^{\circ}$} & PI-B & 51.013 & 39,8 & 0,056 \\
\hline & $\mathrm{PI}-\mathrm{A}$ & 25.176 & 32,3 & 0,149 \\
\hline \multirow[t]{2}{*}{$5^{\circ}$} & PI-B & 81.357 & 50,3 & 0,040 \\
\hline & PI-A & 750 & 1,2 & 0,002 \\
\hline \multirow{2}{*}{$\begin{array}{l}\text { Total diretamente ajustado } \\
\text { entre os quintis }\end{array}$} & PI-B & 198.007 & 46,4 & 0,042 \\
\hline & $\mathrm{PI}-\mathrm{A}$ & 36.911 & 24,3 & 0,075 \\
\hline
\end{tabular}

Baseado no escore de propensão estimado.

Fonte: elaboração própria a partir dos microdados da PNS-2013.

\section{Discussão}

Os resultados obtidos mostraram que mais de $60 \%$ dos clientes de planos de saúde privados e particulares residem na região sudeste do Brasil, onde se concentra a maior parte da rede privada credenciada pelas operadoras de planos de saúde. Essa falta de abrangência geográfica, por si só, já con- tribui com as afirmações de Scheffer \& Bahia (2015) de que os planos privados estão longe de representar uma solução para desafogar o SUS. Além disso, a ocorrência da utilização do SUS, mesmo entre as pessoas vinculadas aos planos de saúde com maior potencial de cobertura, também reforça essa afirmação dos autores. 


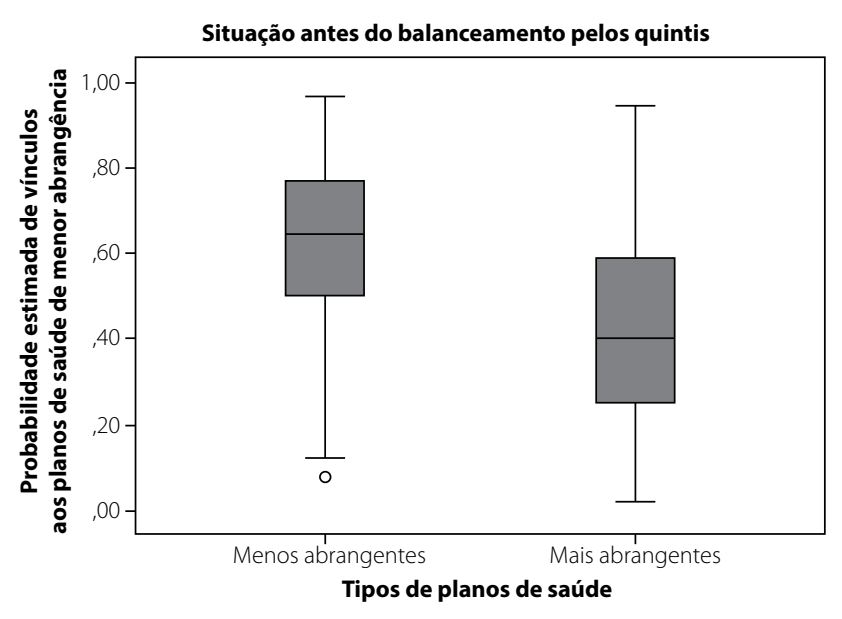

Figura 1. Probalidade estimada do escore de propensão segundo o vínculo ao tipo de plano, Pesquisa Nacional de Saúde (PNS), Brasil, 2013.

Fonte: Elaboração própria dos microdados da PNS-2013.

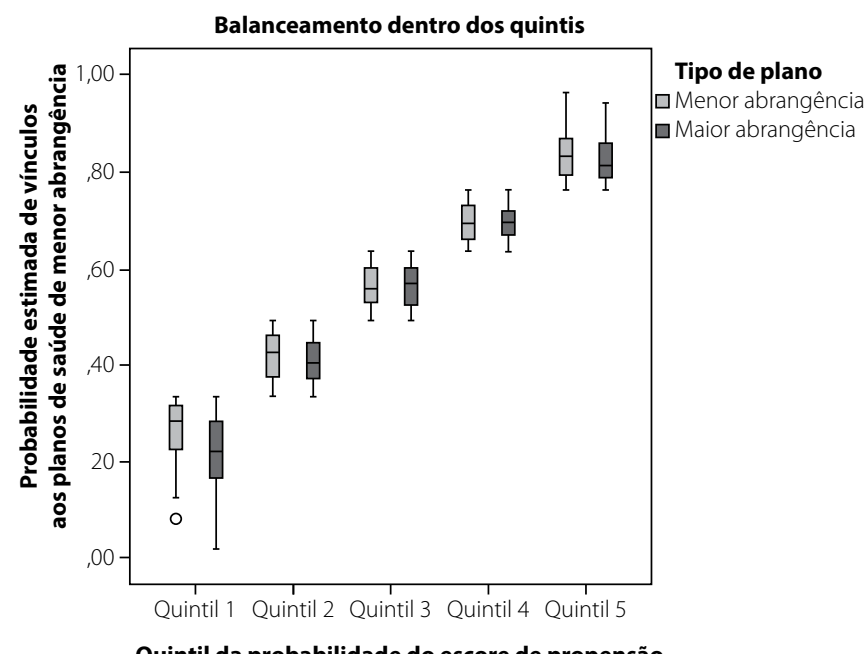

Figura 2. Balanceamento dentro dos quintis da probabilidade estimada do escore de propensão. Pesquisa Nacional de Saúde (PNS), Brasil, 2013.

Fonte: Elaboração própria dos microdados da PNS-2013.

Embora os clientes de planos de saúde com maior renda já contem com maior facilidade de acesso aos serviços de saúde, em concordância com o que asseverou Viana et al. (2015) sobre o melhor acesso aos sistemas a essa parcela da população, era de esperar que não necessitassem recorrer ao SUS, afinal desembolsam quantias mensais relativamente altas, que podem comprometer boa parte de suas rendas, para contar com um serviço em saúde que oferte melhores cuidados e condições de acesso e utilização. Essa utilização serve para mostrar também que, mesmo entre as classes de maior renda per capita, a potencial utilização da rede de serviços do SUS é uma realidade.
Observou-se nos dados brutos, no que tange à população de estudo, que não houve diferença significativa entre o percentual de utilização do SUS para consultas e outros procedimentos, que foi de 15,0\% (IC de 95\%: 13,1\% a 17,1\%), e o percentual de utilização para as internações, que foi de 17,0\% (IC de 95\%: 13,8\% a 20,8\%). Esse resultado contrariou um pouco as expectativas, uma vez que existem, entre as várias modalidades de planos de saúde, muitos que não ofertam aos seus clientes a opção de internação em sua cesta de serviços. São planos apenas ambulatoriais que, em caso de necessidade de internação, como asseveram Barros e Sousa (2016), obrigam sua clientela a recorrer ao SUS ou a pagar diretamente pela internação.

Não existe efetivamente, na prática, uma linha divisória nítida que separe o que é um "plano barato" considerado de menor abrangência de um plano dito "caro" com maior abrangência. Embora tenham sido utilizados critérios técnicos, citados na metodologia, com o intuito de nortear essa separação, ainda assim o estabelecimento da "linha de corte" para o valor de $\mathrm{R} \$ 300,00$ utilizada nessa pesquisa foi parcialmente arbitrário.

Estabelecida essa linha, verificou-se que cerca de metade dos planos individuais pertencia à categoria de planos baratos ou considerados de menor abrangência. Especificamente, encontraram-se 52,9\% (IC de 95\%: 49,7\% a 56,2\%) de vínculos a esses planos (PI-B) e 47,1\% (IC de 95\%: 43,8\% a 50,3\%) de vínculos aos planos ditos "caros" (PI-A).

Ainda que não exista uma localização precisa dessa linha no espaço, é fácil observar, pelas informações contidas no banco de dados da PNS, a existência de uma relação inversa entre a abrangência de um plano de saúde, representada pelo valor da mensalidade, e a busca do atendimento na rede de serviços do SUS por seus respectivos clientes. Esse fato, por já ser esperado, não se constitui propriamente em uma novidade. No entanto, em consonância com essa observação, verificou-se maior utilização da rede de serviços do SUS, tanto para consultas e procedimentos quanto para internações, entre as pessoas vinculadas aos planos cujas mensalidades eram iguais ou inferiores a R\$300,00 (PI-B). Isso está de acordo com o que assevera Scheffer (2015) sobre a maior utilização da rede do SUS que ocorre entre os clientes dos planos ditos "mais populares", ressaltando ainda que, se a justificativa para a legalização da oferta desses tipos de planos no mercado for desafogar o sistema público, eles não seriam capazes de fazê-lo.

Os percentuais brutos de utilização do SUS inicialmente observados ao proceder ao cruzamento de informações da variável desfecho (utilização do SUS) com a variável de exposição (tipo de plano individual), tanto para consultas e outros procedimentos quanto para internações, constituem-se em resultados obtidos baseados nas técnicas tradicionais geralmente empregadas em estudos seccionais. Sendo assim, não se pode descartar a hipótese da ocorrência do confundimen- 
to nesse tipo de análise preliminar. Consequentemente, esse resultado não permite que se possa inferir com segurança sobre a natureza dessa associação. Ou seja, não se pode ser categórico ao afirmar que o vínculo a um plano de menor abrangência se constitui em um incremento ou causa à utilização da rede de serviços do SUS por esses clientes.

Essa utilização da metodologia tradicional não permite que se possa contar com a segurança propiciada pelos estudos randomizados. Porém, ao se trabalhar com a técnica do escore de propensão, "simulou-se" uma situação semelhante à randomização. Isso possibilitou a comparação entre os indivíduos da população de estudo quando eles, em média, possuíam a mesma probabilidade de exposição ou, em outras palavras, o mesmo escore de propensão.

Ao se proceder ao ajuste do escore de propensão pelos quintis para consultas e outros procedimentos nos últimos 15 dias, o resultado global obtido por meio da ponderação dos resultados de cada quintil demonstrou a utilização significativamente maior da rede de serviços do SUS pelos clientes de planos individuais de menor abrangência em relação a essa mesma utilização verificada entre os clientes vinculados aos planos de maior abrangência. Do mesmo modo, essa significância foi verificada no que concerne às internações por 24 horas ou mais ocorridas nos últimos 12 meses.

Cabe ainda ressaltar que a carência de dados mais robustos, que poderiam estar disponibilizados, tanto pela PNS quanto pela ANS, fazem muita falta quando se busca maior consistência nos resultados dos trabalhos acadêmicos. No caso da ANS, por exemplo, há muitas informações relacionadas às operadoras, porém não são encontradas informações referentes aos clientes dos planos. A PNS realizada em 2013 resumiu muito a quantidade de informações sobre a utilização dos sistemas de saúde em relação às pesquisas de 2008 e 2003. Isso demonstra a necessidade de maior interação entre a academia, que produzirá trabalhos com os dados fornecidos, e os órgãos governamentais responsáveis pela coleta de informações.

No entanto, este estudo permitiu que se conclua, pelo fato de ter sido constatada a significância estatística em todos os resultados globais após o ajuste pelo escore de propensão, que há boas sinalizações de que o vínculo a um plano de saúde de menor abrangência se constitui em um incremento, ou causa, à utilização da rede de serviços do SUS por essa clientela. Constituindo-se em uma causa, não são relevantes as considerações de quaisquer propostas que objetivem desafogar o sistema público mediante aprovação, legalização ou criação de sistemas de proteção à saúde, com cobertura limitada ou de pouca abrangência, consequentemente de menor custo mensal, a serem ofertados à população.

\section{Referências bibliográficas}

Azevedo Junior R, Scheffer M, Biancarelli A. Os médicos e os planos de saúde: guia de direitos contra os abusos praticados pelas operadoras. São Paulo: Conselho Regional de Medicina do Estado de São Paulo; 2012.

Bahia L, Cueto M, Benchimol J, Teixeira LA, Cerqueira RC. Pensar o Sistema Único de Saúde do século XXI: entrevista com Lígia Bahia. Hist Ciênc Saúde Manguinhos. 2014;21(1):93-107.

Barros FP, Sousa MF. Equidade: seus conceitos, significações e implicações para o SUS. Saude Soc. 2016;25(1):9-18.

Brasil-ANS. Agência Nacional de Saúde Suplementar (ANS). Sistema de Informações ao Beneficiário; Dados Gerais. 2018. Available from: http:// www.ans.gov.br/perfil-do-setor/dadosgerais. Accessed on: Mar 30, 2018.

Brasil. Constituição da República Federativa do Brasil de 1988. Brasília: Senado, 1988. Available from: http://www.planalto.gov.br/ccivil03/ Constituicao/ Constituicao.htm. Accessed on: Nov 2, 2017.

Hosmer DW, Lemeshow S. Applied Logistic Regression. New York: John Wiley \& Sons; 2002.

IBGE - Instituto Brasileiro de Geografia e Estatística. Pesquisa Nacional de Saúde, 2013: percepção do estado de saúde, estilos de vida e doenças crônicas: Brasil, grandes regiões e unidades da federação. Rio de Janeiro: IBGE; 2014.

Luiz RR, Struchiner CJ. Inferência causal em epidemiologia: o modelo de respostas potenciais. Rio de Janeiro: Editora Fiocruz; 2002. 112p.

Mendes SB. Saúde não tem preço, mas medicina tem custo. Revista JRS. Comunicação. São Paulo. 2016. Available from: https://oglobo.globo. $\mathrm{com} /$ opiniao/saude-nao-tem-preco-mas-medicina-tem-custo19889806f. Accessed on: Dec 15, 2017.

Santos IS, Ugá MAD, Porto SM. O mix público-privado no sistema de saúde brasileiro: financiamento, oferta e utilização de serviços de saúde. Ciênc Saúde Coletiva. 2008:13(5):1431-40.

Scheffer M, Bahia L. Representação política e interesses particulares na saúde: a participação de empresas de planos de saúde no financiamento de campanhas eleitorais em 2014. Relatório de pesquisa, 2015. Available from: http://www.abrasco.org.br/site/wp-content/ uploads/2015/02/Planos-de-Saude-e-Eleicoes-FEV-2015-1.pdf. Accessed on: Feb 8, 2015.

Scheffer M. O capital estrangeiro e a privatização do sistema de saúde brasileiro. Cad Saude Publica. 2015;31(4):663-6.

Sestelo JA, Souza LE, Bahia L. Saúde suplementar no Brasil: revisão crítica da literatura de 2000 a 2010. Saúde Debate. 2014;38(102):607-23.

Silva ZP, Ribeiro MC, Barata RB, Almeida MF. Perfil sociodemográfico e padrão de utilização dos serviços de saúde do Sistema Único de Saúde (SUS), 2003- 2008. Ciênc Saúde Coletiva. 2011;16(9):3807-16.

Souza-Júnior PR, Freitas MP, Antonaci GA, Szwarcwald CL. Desenho da amostra da Pesquisa Nacional de Saúde 2013. Epidemiol Serv Saúde. 2015;24(2):207-16

Viana KP, Brito AS, Rodrigues CS, Luiz RR. Access to continued-use medication among older adults. Br Rev. Saúde Publica. 2015;49:14 